\title{
A microwave kinetic inductance camera for sub/millimeter astrophysics
}

Jason Glenn, Peter K. Day, Matt Ferry, Jiansong Gao, Sunil R. Golwala, et al.

Jason Glenn, Peter K. Day, Matt Ferry, Jiansong Gao, Sunil R. Golwala, Shwetank Kumar, Henry G. LeDuc, Philip R. Maloney, Benjamin A. Mazin, Hien Nguyen, Omid Noroozian, Jack Sayers, James Schlaerth, John E. Vaillancourt, Anastasios Vayokanis, Jonas Zmuidzinas, "A microwave kinetic inductance camera for sub/millimeter astrophysics," Proc. SPIE 7020, Millimeter and Submillimeter Detectors and Instrumentation for Astronomy IV, 70200B (18 July 2008); doi: 10.1117/12.790050

Event: SPIE Astronomical Telescopes + Instrumentation, 2008, Marseille, France 


\title{
A microwave kinetic inductance camera for sub/millimeter astrophysics
}

\author{
Jason Glenn*a, Peter K. Day ${ }^{\mathrm{b}}$, Matt Ferry ${ }^{\mathrm{c}}$, Jiansong Gao ${ }^{\mathrm{c}}$, Sunil R. Golwala ${ }^{\mathrm{c}}$, Shwetank Kumar ${ }^{\mathrm{c}}$, \\ Henry G. LeDuc ${ }^{b}$, Philip R. Maloney ${ }^{\mathrm{a}}$, Benjamin A. Mazin ${ }^{\mathrm{b}}$, Hien Nguyen ${ }^{\mathrm{b}}$, Omid Noroozian $^{\mathrm{c}}$, Jack \\ Sayers $^{\mathrm{c}}$, James Schlaerth ${ }^{\mathrm{a}}$, John E. Vaillancourt ${ }^{\mathrm{c}}$, Anastasios Vayokanis ${ }^{\mathrm{c}}$, Jonas Zmuidzinas ${ }^{\mathrm{b}, \mathrm{c}}$ \\ ${ }^{a}$ Department of Astrophysical and Planetary Sciences, CASA 389-UCB, University of Colorado, \\ Boulder, CO 80309; \\ bet Propulsion Lab, 4800 Oak Grove, Pasadena, CA 91107; \\ ${ }^{\mathrm{c}}$ Dept. of Physics, Mathematics, and Astronomy, California Institute of Technology, 1200 East \\ California Blvd., Pasadena, CA 91125 \\ *jglenn@casa.colorado.edu
}

\begin{abstract}
The MKID Camera is a millimeter/submillimeter instrument being built for astronomical observations from the Caltech Submillimeter Observatory. It utilizes microwave kinetic inductance detectors, which are rapidly achieving near-BLIP sensitivity for ground-based observations, and a software-defined radio readout technique for elegant multiplexing of a large number of detectors. The Camera will have 592 pixels distributed over 16 tiles in the focal plane, with four colors per pixel matched to the $750 \mu \mathrm{m}, 850 \mu \mathrm{m}$, and $1.0-1.5 \mathrm{~mm}$ (split in two) atmospheric transmission windows. As a precursor to building the full-up camera and to enable ongoing detector testing, we have built a DemoCam comprised of a 16-pixel MKID array with which we have made preliminary astronomical observations. These observations demonstrate the viability of MKIDs for submillimeter astronomy, provide insight into systematic design issues that must be considered for MKID-based instruments, and they are the first astronomical observations with antenna-coupled superconducting detectors. In this paper, we describe the basic systems and specifications of the MKID Camera, we describe our DemoCam observations, and we comment on the status of submillimeter MKID sensitivities.
\end{abstract}

Keywords: Submillimeter astronomy, Microwave Kinetic Inductance Detectors, software defined radio

\section{INTRODUCTION}

Two primary science drivers combined with the technological readiness of Microwave Kinetic Inductance Detectors (MKIDs; Section 2) have motivated our MKID Camera. First, in the wake of the discovery of the cosmic far-infrared background with $\mathrm{COBE}^{1,2}$, hundreds of submillimeter galaxies (galaxies identified by the predominance of their submillimeter-wavelength thermal dust emission) have been detected with bolometer array cameras. By combining submillimeter observations with observations at other wavebands, the astrophysics of the galaxies have begun to be revealed and their cosmological importance, in terms of developing a comprehensive galaxy formation model, has become evident. SCUBA-2 is poised to detect many new dusty galaxies over a range of redshifts, and the Herschel Space Observatory will have photometric coverage from $60 \mu \mathrm{m}$ to $670 \mu \mathrm{m}$. The MKID Camera, with spectral coverage from $750 \mu \mathrm{m}$ to $1.5 \mathrm{~mm}$, will complement these instruments for continuous coverage of SEDs over most of the interstellar thermal dust emission spectrum. Second, large surveys of the Galactic plane are coming of age. Panchromatic surveys will reveal the processes of the formation and evolution of molecular clouds, from the diffuse interstellar medium to star formation. The MKID Camera will observe optically thin dust emission for measurements of cloud core masses.

The MKID Camera is a new instrument being constructed for the Caltech Submillimeter Observatory (CSO). It will perform simultaneous imaging in four wavelength bands: $1.3 \mathrm{~mm}, 1.05 \mathrm{~mm}, 860 \mu \mathrm{m}$, and $740 \mu \mathrm{m}$. This will be accomplished using a focal plane array of MKIDs operating at a base temperature of $230 \mathrm{mK}$, with a completely closedcycle cryogenic system combining a He sorption refrigerator and a pulse tube cooler. The array will have $24 \times 24$ spatial pixels, and each pixel will simultaneously respond to the four wavelength bands; thus, the total detector count will be 24

Millimeter and Submillimeter Detectors and Instrumentation for Astronomy IV edited by William D. Duncan, Wayne S. Holland, Stafford Withington, Jonas Zmuidzinas Proc. of SPIE Vol. 7020, 70200B, (2008) · 0277-786X/08/\$18 · doi: 10.1117/12.790050

Proc. of SPIE Vol. 7020 70200B-1 
$\times 24 \times 4=2304$. A software defined radio technique that will likely find applications in other highly multiplexed MKID and microwave SQUID-based instruments will be used to read out the MKIDs.

To demonstrate the viability of MKIDs for astronomical observations, to serve as a testbed for characterizing systemslevel issues to be reckoned with in using MKIDs for submillimeter astronomy, and for exploring MKID design space for detector parameter optimization, we built the MKID Democam. It is comprised of 16 two-color $(860 \mu \mathrm{m}$ and $1.25 \mathrm{~mm})$ MKIDs and a trial electronics readout system. With it, we have obtained observations at the CSO of planets and ultracompact HII regions; these are described in Section 6. In Section 2, we describe the operational principles of MKIDs; Section 3 describes the readout electronics; the optical design and cryogenics are described in Section 4; and Section 5 briefly describes the data pipeline.

\section{MKIDS}

Microwave Kinetic Inductance Detectors ${ }^{3}$ (MKIDs) are high-Q superconducting resonators. Although superconductors exhibit zero DC resistance, they still have nonzero surface impedance to AC currents. This impedance is composed of a real resistive part and an imaginary inductive part. Absorbed radiation breaks Cooper pairs, altering the surface impedance, thereby changing the resonance frequency of the MKID circuit and introducing a phase shift (Figure 1). For small optical loads, this response is linear, and thus useful for astronomical observations. Changes in an MKID resonant frequency (or phase) due to absorption of radiation are detected by sending an on-resonance probe signal through a capacitively coupled coplanar waveguide feedline. In practice, many different resonators with slightly different resonant frequencies can be coupled to the same feedline, allowing for elegant frequency multiplexing with negligible crosstalk. A cryogenic HEMT amplifies the signals for room-temperature demodulation of the transmitted amplitude and phase. Since single HEMTs have bandwidths sufficient to amplify thousands of MKID probe signals, all of the complex electronics can reside at room temperature.
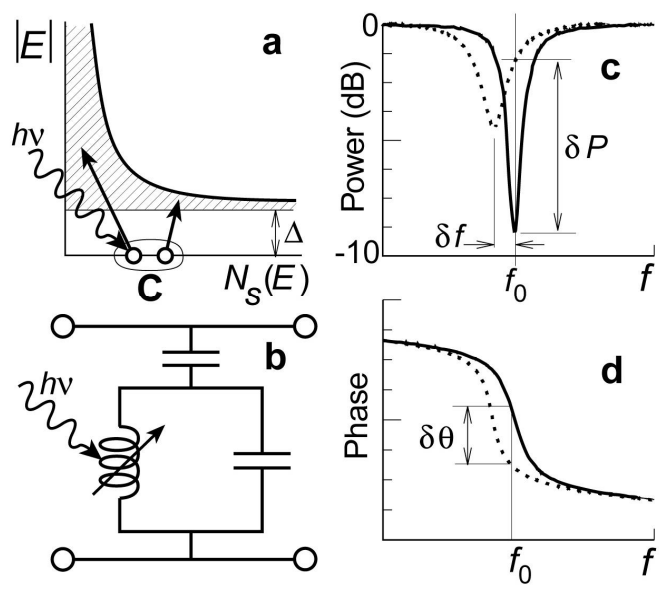

Fig. 1. Operational principle of a MKIDs. (a) Incoming photons with energy greater than $2 \Delta$ (corresponding to $v>90 \mathrm{GHz}$ for Al MKIDs) break Cooper pairs leading to quasiparticles with a distribution of energies. (b) An equivalent circuit of an MKID showing its variable inductance and capacitive coupling to a probe signal feed line. (c) Alteration of the MKID surface impedance from the creation of quasiparticles shifts the resonant frequency, thereby reducing the amplitude of the MKID's response to a fixed-frequency probe signal. (d) Similarly, a phase shift is introduced.

We couple optical radiation to the MKIDs via slot-dipole phased-array antennas. A demonstration 16-pixel array similar to the one used in the DemoCam (Section 6) is shown in Figure 2. This type of antenna ${ }^{4,5}$ produces a narrow beam pattern by combining signals from an array of slots in phase using niobium microstrip transmission lines. The optical power exits the phased array for each pixel on microstrip and is coupled to MKIDs after passing through in-line banddefining filters. The filters are compact, lumped-element, three-stage $L C$ networks that have sharp band edges and no 
high-frequency harmonic leaks ${ }^{5}$. Response to high-frequency thermal infrared radiation is further suppressed by the antenna feed network, which becomes extremely lossy above the $700 \mathrm{GHz}$ gap frequency of $\mathrm{Nb}$. Since the filters have high out-of-band impedance, they can be put in parallel to obtain several colors from a single spatial pixel.

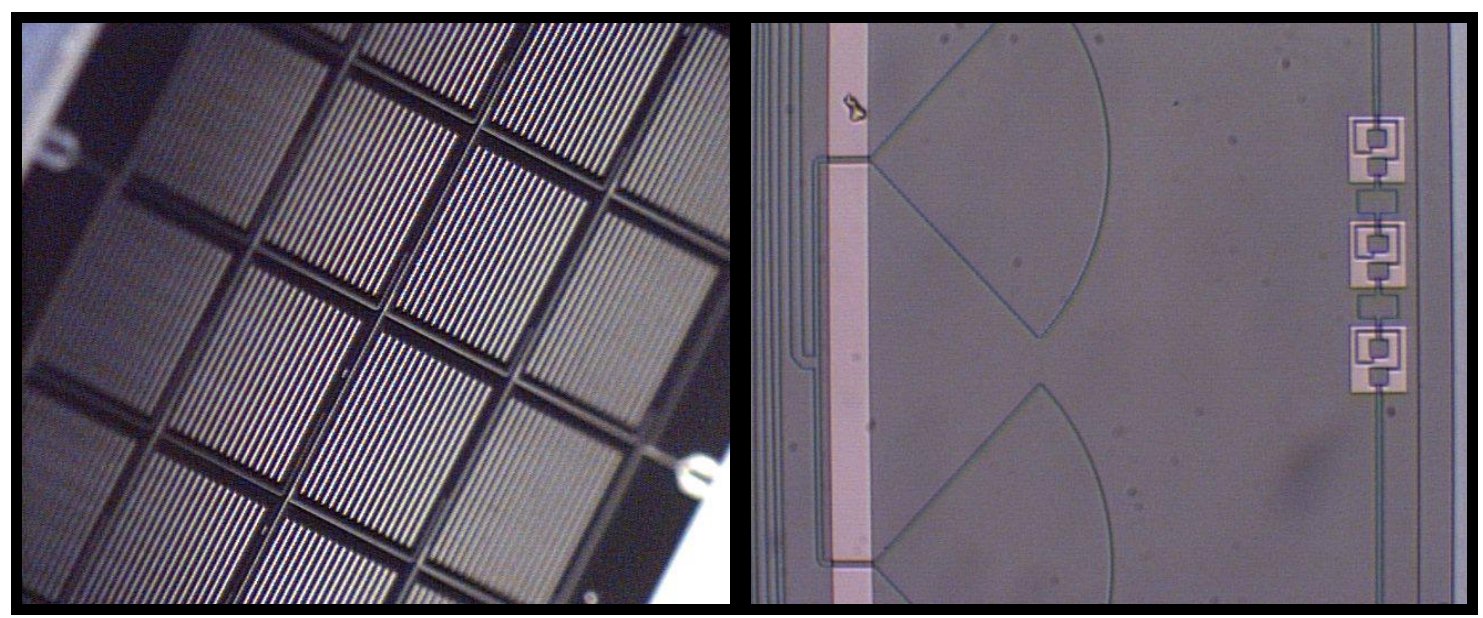

Fig. 2. (Left) MKID 16-pixel detector array (with a 4-detector $\times$ 4-detector format). The linear patterns in each pixel are the (single-polarization) slot-dipole antennas. The pixels are square, $3.3 \mathrm{~mm}$ on a side. (Right) A close-up of a region of one of the pixels showing a portion of a (vertical) antenna slot with taps and stub capacitors on the left and bandpass-defining filter elements on the right.

\section{ELECTRONICS}

The readout of the MKID Camera will be done with room temperature electronics, an enormous advantage compared to other detector technologies, such as high-impedance semiconductor bolometers, which require $\sim 100-\mathrm{K}$ JFET amplifiers, or transition-edge sensors, which require cryogenic SQUIDs for every pixel. By using very fast A/D converters to digitize large chunks of bandwidth, we can take advantage of the flexibility of fast FPGAs and advanced signal processing techniques to extract the desired signals ${ }^{6}$.

A single-resonator readout circuit for an MKID would be set up as follows (see Figure 3). A microwave frequency synthesizer creates a signal at the resonator frequency $(2-6 \mathrm{GHz})$ which is divided by a splitter. One copy, the probe signal, is sent through the cryostat, resonator, and amplifiers, and into the RF port of the down-converting IQ mixer. The other copy is used to drive the LO port of this mixer. This is known as homodyne mixing, since the same signal is used for the LO and the RF. The output of the down-converting mixer gives the in-phase $(I)$ and quadrature $(Q)$ parts of the signal, from which the amplitude and phase changes introduced by the resonator can be derived.

Since the off-resonance transmission of the MKIDs is essentially unity, we can multiplex the readout of a large number of resonators using a single probe signal that contains a comb of frequency tones matched to each MKID; this comb will be generated by dual fast DACs which will drive the IQ ports of an up-converting mixer. In our present concept, the readout electronics will also be partitioned into 16 modules, with each module reading out a single tile of 144 resonators.

The multiplexed probe signal will be generated at baseband frequency $(0-200 \mathrm{MHz})$ using high-speed, large-bandwidth DACs (D/As) to play back a predefined, easily modified waveform stored in memory. Easy modification of the waveform is required because the resonant frequencies of the MKIDs change as a function of observing conditions (i.e., telescope elevation angle due to the Earth's magnetic field or atmospheric water vapor content). The baseband DAC (D/A) signals then will be upconverted to the microwave band and sent into the cryostat to excite the resonators. A frequency step size of $1 \mathrm{kHz}$ or less in the (pre-upconverted) waveforms is necessary to center them on the MKID resonances for $\mathrm{Q} \geq 10^{4}$. By using a dual-DAC scheme with an up-converting IQ mixer and a similar scheme with dual ADCs (A/Ds) on the output side, the need for single-sideband conversion is avoided. An FPGA core will be used as a narrow band channelizer to digitally process these data, essentially demodulating it at each comb frequency to determine 
the response of each resonator. This will likely involve some combination of FFTs and digital-to-digital converters. The demodulated signal for each resonator will be stored to disk at a data rate of approximately $100-200 \mathrm{~Hz}$, (a minimum of $100 \mathrm{samples} / \mathrm{sec}$ per channel), yielding a total data rate of 1.2 Mbytes per second (16 modules $\times 128$ channels per module $\times 100$ samples per second $\times 6$ bytes/sample).

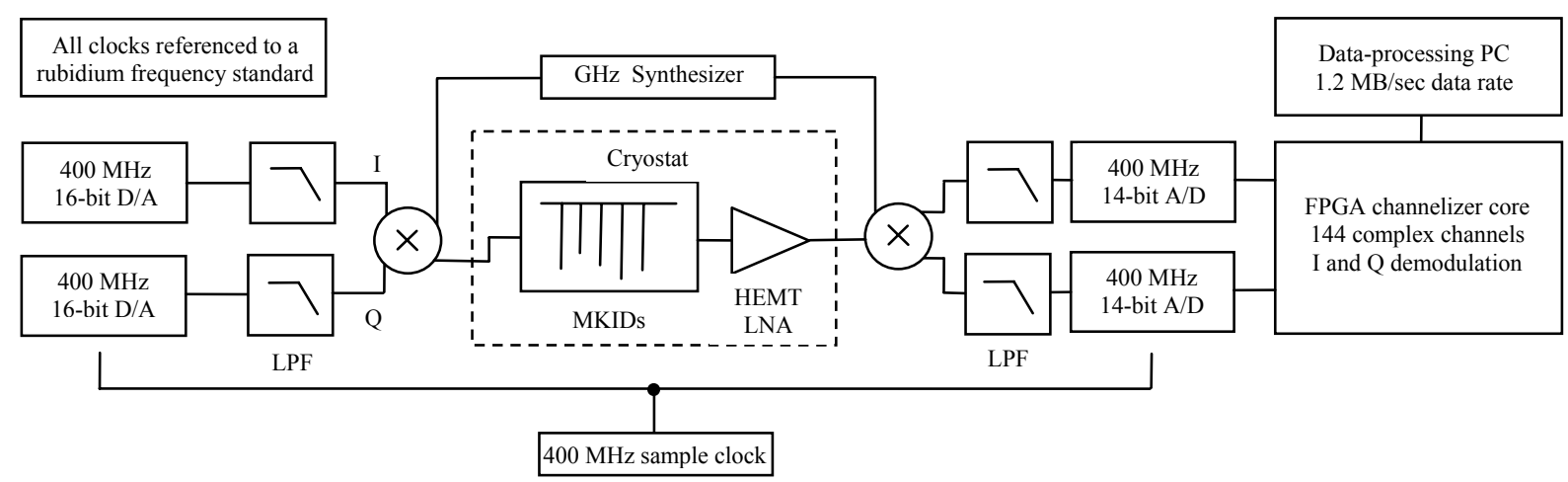

Fig. 3. MKID Camera electronics schematic. The electronics will be comprised of sixteen modules, each of which will read out 144 channels: one tile of 36 4-color MKIDs. Each module will have dual $400 \mathrm{MHz} 16$-bit D/As, upconversion and downconversion via IQ mixers, and $400 \mathrm{MHz}, 14-\mathrm{bit} \mathrm{A} / \mathrm{Ds}$, and an FPGA channelizer core.

Key specifications of the electronics system are summarized in Table 1. Some considerations are: 1) the Camera performance should be BLIP limited (see Section 6), 2) the readout electronics noise should be limited by the cryogenic (HEMT) amplifier noise, 3) 14-bit, 400 megasample per second A/Ds with a signal-to-noise ratio greater than $56 \mathrm{~dB}$ likely will required, and the D/As and phase noise of the frequency synthesizer should not add significant noise to the system.

Table. 1. MKID readout electronics specifications required for the MKID Camera.

\begin{tabular}{|l|c|}
\hline \multicolumn{1}{|c|}{ Characteristic/Component } & Specification \\
\hline Number of spatial pixels & 576 \\
Number of colors (resonators) per pixel & 4 \\
Number of resonators per tile & 144 \\
Number of modules (one per tile) & 16 \\
Number of channels per module & 144 \\
Carrier-to-noise ratio per channel (minimum) & $123 \mathrm{dBc} / \mathrm{Hz}$ \\
Resonator tile input/output frequency range & $2-6 \mathrm{GHz}$ \\
Bandwidth per tile & $400 \mathrm{MHz}$ \\
Output data format, word size & Complex integer, $2 \times 24 \mathrm{bits}$ \\
Data rate & $1.2 \mathrm{MB} / \mathrm{second}$ \\
D/A frequency step size & $1 \mathrm{kHz}$ \\
\hline
\end{tabular}




\section{CRYOSTAT}

A schematic of the MKID Camera cryostat is shown in Figure 4. The cryostat is very compact with a length of just over one meter and a diameter of 0.6 meters. It will mount on the CSO Bolocam optics box, which has two flat folding mirrors and an off-axis ellipsoidal mirror that translate the Cassegrain focus into the cryostat. Refrigeration of the MKID focal plane array and optics to cryogenic temperatures will be accomplished with a Cryomech PT-415 cryocooler. This is a two-stage pulse-tube cooler. The first stage, with $40 \mathrm{~W}$ of heat lift at $40 \mathrm{~K}$, will be used to cool radiation shields and intercept the heat load from wiring leading from the room-temperature vacuum shell to the subKelvin stage. The second stage will cool the He sorption fridge condensation stage to approximately $4 \mathrm{~K}$, making use of a 1.5 -W heat lift - sufficient for He fridge recycling. The 3 -stage Chase Cryogenics ${ }^{\mathrm{TM}}$ refrigerator $\left({ }^{4} \mathrm{He} /{ }^{\beta} \mathrm{He} /{ }^{\beta} \mathrm{He}\right)$ supports a heat load of $3-4 \mu \mathrm{W}$ at $250 \mathrm{mK}$.

At the top of the vacuum vessel, ballast tanks for the pulse tube cooler are visible along with the remote valve. Cylindrical G-10 fiberglass standoffs support the 40-K and 4-K stages. As seen in the upper left-central portion of the schematic, the pulse tube cooler descends to the 4-K work surface. The largest G-10 cylinder houses the He refrigerator, which is recessed into the $40-\mathrm{K}$ space to minimize the volume of the cryostat. Toward the bottom of the cryostat is a cylindrical mumetal magnetic shield (which will provide $35 \mathrm{~dB}$ of attenuation), with a conical tubulation for the radiation path, that houses the MKID focal plane and the field lens.

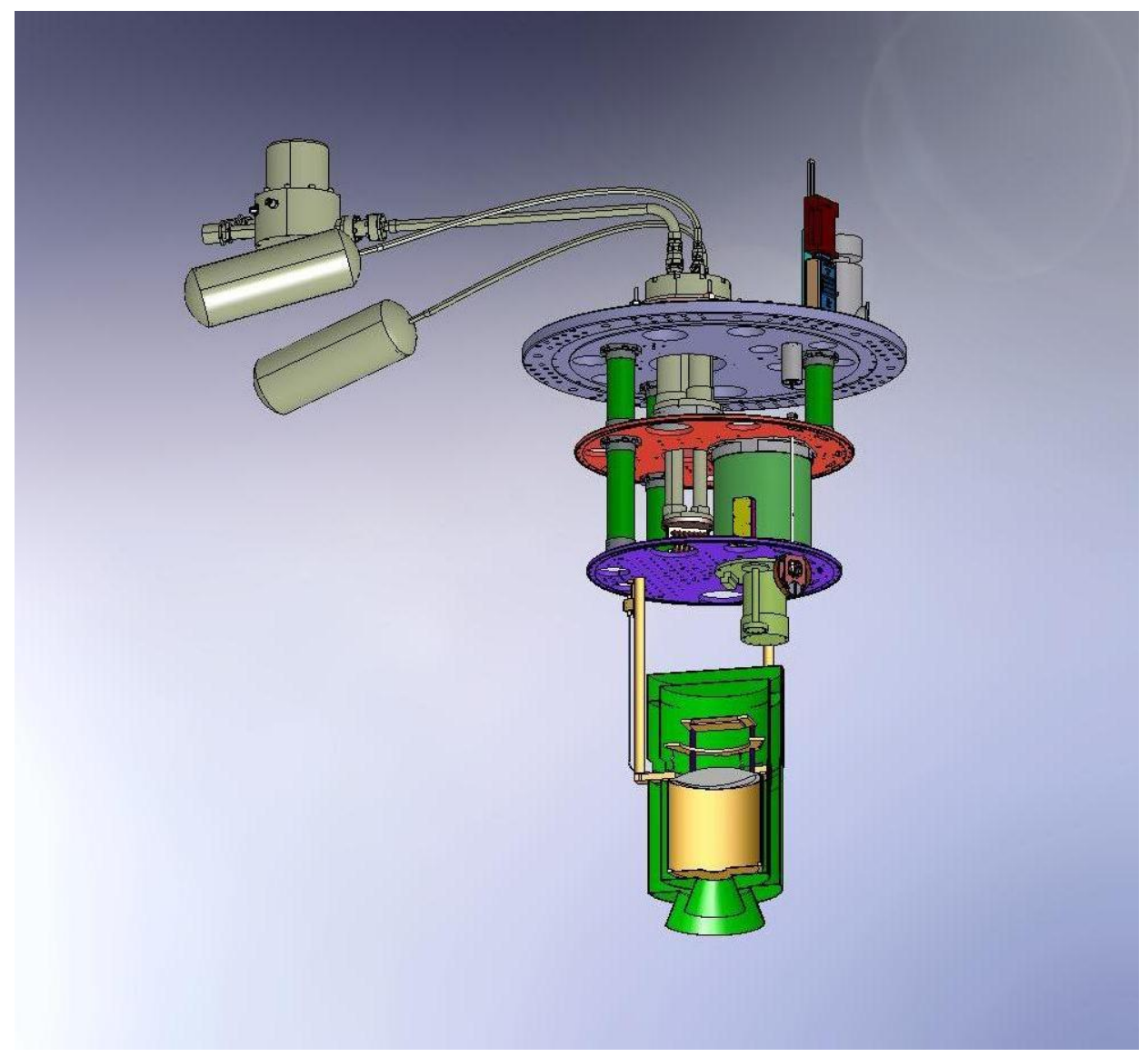

Fig. 4. Schematic of the MKID Camera Cryostat (drawing courtesy High Precision Devices, Inc., Boulder, CO). The radiation shields have been removed for clarity and the optics are shown in a cutaway view. The cryostat is downwardlooking and will mount to the CSO Bolocam optics box in nearly this orientation. The components are described in the text. In the actual assembly the (cylindrical) ballast tanks, visible in the upper left, will be mounted directly on top of the cryostat. 
MKIDs have advantages that allow for the simple, compact design. With the slot-dipole antennas, bandpass filters, and resonators integrated on single silicon wafers (16 wafers, or tiles, comprise the entire focal plane detector array), no stacking of feedhorns or backshorts (and their attendant tight alignment tolerances) are required. Furthermore, because the resonator Qs are so large $\left(10^{4}\right)$, many resonators can be coupled to a small number of HEMT amplifiers. It would be possible to use just two HEMTs for the entire focal plane array with 2304 channels; however, for convenience sixteen HEMTs will likely be used (one HEMT per tile). Thus, the entire wiring harness will consist of 32 rigid coaxial cables and a housekeeping (thermometry and HEMT bias) cable. In Figure 3, the HEMTs are omitted (they will reside in the 40-K space, but they will be heat stationed to the 4-K plate) and only two of the eight coaxial cables are shown descending to the mumetal cylinder (one in the foreground and one in the background).

The detailed optical design has not been completed - it awaits a thorough analysis of coupling efficiency to the slotdipole antennas as a function of $\mathrm{f} / \#$. However, some elements of the system have been established and the design is extremely simple and entirely on-axis within the cryostat. It is based upon the Bolocam optical design ${ }^{7,8}$, which provides Strehl ratios $>0.95$ across the entire 8 arcminutes field of view at a wavelength of $1.1 \mathrm{~mm}$. The cryostat will feature an optical vacuum window made of Zotefoam ${ }^{\mathrm{TM}}$ or ultra-high molecular weight polyethylene. Light will pass through the window and encounter a $40-\mathrm{K}$ filter of PTFE ( $\sim 2 \mathrm{~cm}$ thick) anti-reflection coated with Zitex ${ }^{\mathrm{TM}}$. The window to the 4-K space will consist of anti-reflection coated PTFE surrounding a 1-mm thick piece of Fluorogold ${ }^{\mathrm{TM}}$ (or an $18 \mathrm{~cm}^{-1}$ lowpass metal-mesh filter) to eliminate any high frequency light from entering the 4-K volume. Band-defining filters will be on the devices themselves, so optical filters are only used for eliminating out-of-band far-infrared radiation. Following the filter stack, an image of the telescope primary mirror will be formed at a 4-K Lyot stop. Light is then focused through a single HDPE or PTFE lens onto the detector plane. This lens changes the $\mathrm{f} / \#$ of the incoming beam from approximately $f / 7$ to $f / 2$ or $f / 3$ for coupling to the antennas.

\section{DATA PIPELINE}

We will adopt the mature, flexible Bolocam software package $e^{8,9,10,11}$ for the MKID Camera with minimal modifications. This both minimizes development and debugging time and provides users of Bolocam and the MKID Camera with a common interface and software continuity.

The Bolocam pipeline acquires telescope pointing and timing information, merges it with time-stamped data from all the bolometers, and writes it in the self-describing, flexible netCDF format (http://www.unidata.ucar.edu/software/netcdf/). While new software will have to be written to perform this merging step for MKID Camera due to the very different camera data acquisition system, the telescope interface portions of the Bolocam merging code can be reused. Merged data are processed via a flexible, configurable IDL pipeline. Error checking is performed and the data are "cleaned" to remove correlated sky emission. Two sky removal techniques, principal component analysis and spatial polynomial removal, have been implemented. The time stream data are then mapped, correcting for pointing offsets and optical distortion, and calibrated. An iterative mapping algorithm is useful in bright fields to remove artifacts arising from contamination of the sky estimate by astronomical signal. A simplified, automated version of the pipeline produces firstcut maps within minutes of completing an observation. This IDL-based pipeline will be not so much modified as expanded to accommodate MKID Camera. Because the netCDF format is self-describing, increasing the pixel count and generalizing to four colors is straightforward. New modules to make use of spectral sky subtraction, or to do joint spectral and spatial sky subtraction, will be incorporated into the pipeline.

\section{DEMOCAM: PROOF OF CONCEPT AND SENSITIVITY}

\subsection{Description and laboratory characterization}

The DemoCam utilizes a compact liquid helium dewar, with a $230 \mathrm{mK}$ (well below the $\mathrm{T}_{\mathrm{c}}$ of the $\mathrm{Al}$ resonators) He sorption refrigerator, to house an array of 32 MKIDs (16 2-color pixels) and an optical system similar to Bolocam's to 
match it to the Bolocam optics box and the CSO. The cryogenic optics were comprised of a single PTFE field lens to achieve the desired plate scale and a dielectric-only filter stack of PTFE and Fluorogold ${ }^{\mathrm{TM}}$, all anti-reflection coated with Zitex.

Lithographed phased arrays of slot dipole antennas couple radiation incident on the focal plane to the hybrid $\mathrm{Al} / \mathrm{Nb}$ MKIDs: sixteen slot antennas are coadded in-phase to produce a signal in a microstrip feedline. The slot antennas define the beam shape in the far field; their beams are well approximated by uniformly illuminated squares $3.3 \mathrm{~mm} \times 3.3 \mathrm{~mm}$ in size. The signal is then passed through in-line lumped-element bandpass filters and coupled to resonators via a coplanar waveguide with a $6 \mu \mathrm{m}$ center strip and $2 \mu \mathrm{m}$ slots, and approximately $4 \mathrm{~mm}$ long. Thus, there is no need for banddefining optical filters; the necessary bandpasses were achieved solely by on-chip filters (Figure 5), with dielectrics for infrared blocking. Metal-mesh low-pass filters may be used in the MKID Camera for redundancy. The resonant frequencies of the detectors were separated by approximately $10 \mathrm{MHz}$ (starting at $7.5 \mathrm{GHz}$ ).

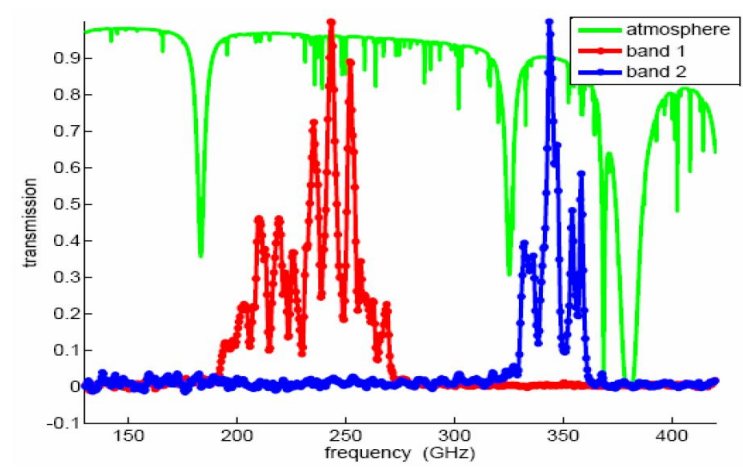

Fig. 5. Spectra of the bandpass filters used in the DemoCam obtained with a Fourier transform spectrometer superposed on a model atmospheric transmission curve. Bands 1 and 2 correspond approximately to the MKID Camera $860 \mu \mathrm{m}$ and 1 $\mathrm{mm}$ long bands. The ringing in the bandpasses likely arises from the MKID circuits, not the dielectric filter stack. Its origin is being investigated so that it can be eliminated.

\subsection{Engineering run results, achieved sensitivity, and implications for MKID Camera sensitivity}

First light was obtained with a raster scan observation of Jupiter using two resonators ${ }^{12}$ (Figure 6). Magnetic shielding was minimal; consequently, the MKIDs were very sensitive to small changes in magnetic fields at the array resulting from telescope motion in the static magnetic field of the Earth, most likely due to trapped magnetic flux in the detectors. Application of an azimuth/elevation linear fit removed the magnetic field effects (drift scans - in which the telescope was held stationary and observations were performed as the sky drifted by - were unaffected by the magnetic field). The MKIDs could be used under a variety of atmospheric loading conditions, differentiating them from transition-edge sensors which can switch from superconducting to normal states under heavy atmospheric loading.

From observations of planets and the G34.3 ultracompact HII region, the noise-equivalent flux densities (NEFDs) were found to be $1.3 \mathrm{Jy} \mathrm{s}^{1 / 2}$ at $1.25 \mathrm{~mm}$ and $8 \mathrm{Jy} \mathrm{s}^{1 / 2}$ at $850 \mu \mathrm{m}$ in raster-scan mode and $1.6 \mathrm{Jy} \mathrm{s}^{1 / 2}$ and $14 \mathrm{Jy} \mathrm{s}{ }^{1 / 2}$, respectively, in drift-scan mode (the focal plane $\mathrm{f} / \#$ was optimized for the $1.25 \mathrm{~mm}$ band). There is a potential advantage in sky subtraction using two colors; however, we did not find this to be the case, likely because of the worse responsivity of the $850 \mu \mathrm{m}$ band. This will be retested in future observing runs.

The sensitivity achieved in the engineering run was approximately 20 times worse than the expected background limit (see below). There are several factors that contributed to this: 1) It likely that the fractional volume of the devices in which quasiparticles are created by pair breaking is approximately four times smaller than expected from laboratory measurements, likely because the device is thicker than anticipated, which reduced the kinetic inductance response. 2) The MKIDs were read out with two separate schemes: one in which the amplitude shift of the demodulated signal was measured and the other in which the phase shift was measured - thus, there were two separate measures of response. The resonators exhibited high phase noise, so that amplitude readout, which inherently has four times poorer 
responsivity than phase readout, had to be used (in general, the phase response has higher noise at low frequencies ${ }^{13}$ ). However, new resonator designs with larger gap widths between the resonators and the coplanar waveguide carrying the excitation signals and an interdigitated capacitor design have been lab-tested and shown to have dramatically reduced phase noise. This will likely enable us to use phase readout rather than amplitude readout in the MKID Camera to take advantage of the greater phase responsivity. 3) The MKID resonators (Qs) were not well matched to the atmospheric loading, which will be remedied for the MKID Camera. A further improvement being implemented for the MKID Camera is that magnetic susceptibility is being reduced substantially with magnetic shielding and by perforating the detector ground plane to pin magnetic flux that would otherwise be trapped in the MKID resonators.
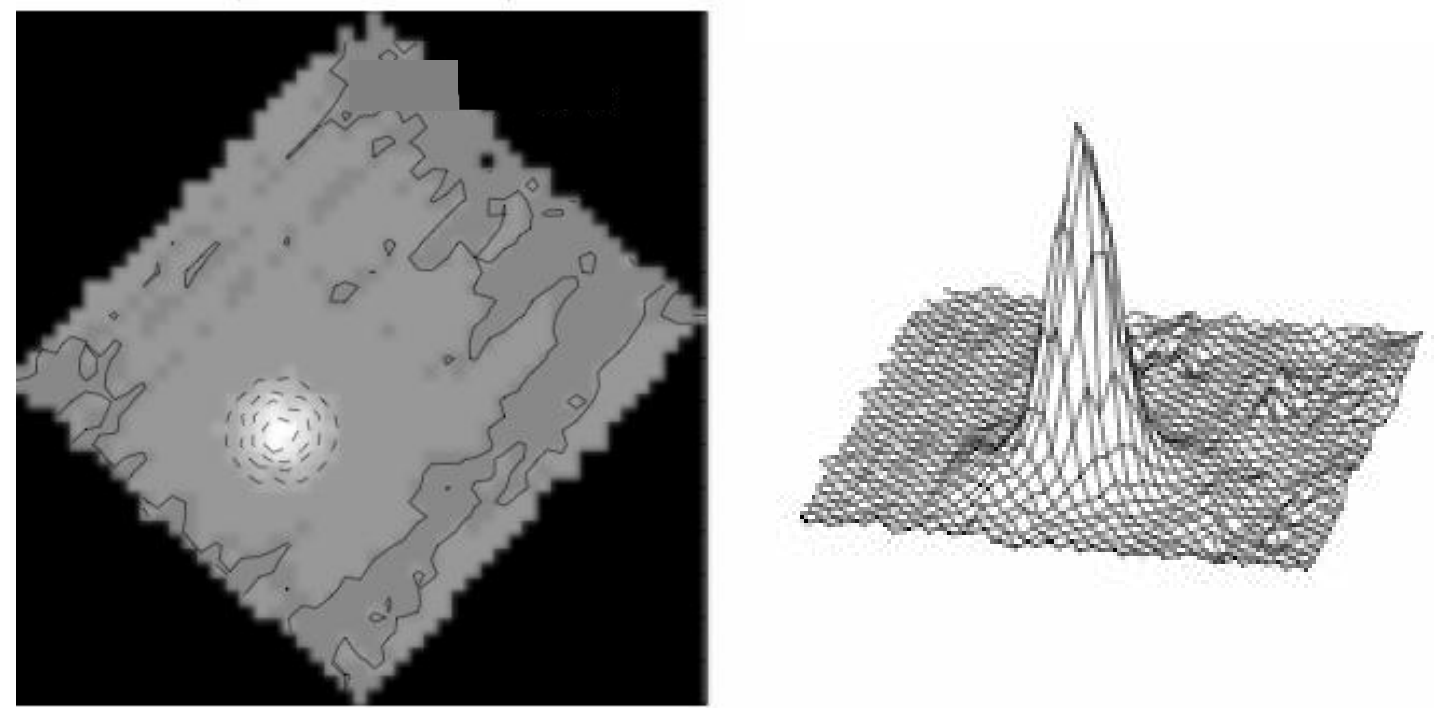

Fig. 6. (Left) A $1.1 \mathrm{~mm}$ grey-scale image with contours of Jupiter obtained with one resonator in MKID Democam. The image is 8 arcminutes on a side. (Right) A three-dimensional projection of the Jupiter image. The beam has a circular cross-section and low sidelobes (sky emission has not been subtracted in this image).

Ideally, the noise performance of the MKID camera should be limited by the random arrival of background photons (BLIP limited). The background photon power for a single-moded single-polarization detector is equal to $\mathrm{P}_{\text {submm }}=$ $\eta \mathrm{k}_{\mathrm{B}} \mathrm{T}_{\text {load }} \Delta v$ where $\eta$ is the optical efficiency, $\mathrm{k}_{\mathrm{B}}$ is Boltzmann's constant, $\mathrm{T}_{\text {load }}$ is the background load, and $\Delta v$ is the spectral bandwidth. In Table 2 we list the design band centers and widths for the four MKID Camera bands, as well as the resulting values of $\mathrm{P}_{\text {submm, }}$ accounting for telescope as well as atmospheric loading for the median value of precipitable water vapor at Mauna Kea. We also give the photon noise equivalent power, or NEP, including both the random and photon-bunching terms. The improvements in MKID noise achieved since the DemoCam engineering run indicate that the MKID camera should be able to reach the background limit.

\section{DISCUSSION AND SUMMARY}

Microwave kinetic inductance detectors have matured considerably in the last several years, to the point that fabrication of arrays numbering in the thousands is possible and performance is approaching BLIP for observations from Mauna Kea for the long-submillimeter bands and $1 \mathrm{~mm}$ band $\left(\sim 10^{-16} \mathrm{~W} \mathrm{~Hz}^{-1 / 2}\right)$. MKIDs provide for elegant multiplexing with minimal cryogenic electronics and emerging flexible software-defined radio readouts. Some development effort is required for the MKID Camera readout, both in hardware - board design and fabrication - and channelization firmware, but both are feasible and the development effort is likely to pay off for other MKID applications across the electromagnetic spectrum (and possibly for dark matter detection) and microwave SQUIDs. Furthermore, computing 
speed will continue to increase and component costs will likely decrease in the coming years, providing further impetus for this digital solution (as opposed to an all-analog demodulation scheme).

We have presented a design for a millimeter/submillimeter MKID camera for the CSO for astronomical observations, notably observations of star formation in the Milky Way and observations of distant star-forming galaxies important for understanding galaxy evolution. The Camera incorporates several new technologies, which simplify its design compared to previous generations of large focal-plane-array millimeter and submillimeter cameras. Among these are MKIDs, bandpass filters, and slot-dipole antennas all integrated on single chips. The compact optical design builds upon the on-axis cryogenic design of Bolocam, but simplified by obviating optical bandpass filters. Some of the key technologies have been demonstrated in the DemoCam testbed with observations from the CSO. These observations represent astronomical first light for superconducting detectors wedded to slot-dipole antennas. They demonstrated the viability of MKIDs for astronomical observations and highlighted systematic issues that need to be addressed, chiefly magnetic shielding around the focal plane and reduced susceptibility to magnetic fields by the MKIDs themselves.

Table. 2. MKID bands: frequencies, atmospheric loading under typical low-frequency (weather-multiplexed) observing conditions at the CSO, and the BLIP-only component of the NEP.

\begin{tabular}{|c|c|c|c|c|}
\hline Band & $\begin{array}{c}v_{0} \\
(\mathrm{GHz})\end{array}$ & $\begin{array}{c}\Delta v \\
(\mathrm{GHz})\end{array}$ & $\begin{array}{c}\mathrm{P} \\
(\mathrm{pW})\end{array}$ & $\begin{array}{c}\mathrm{NEP}_{\mathrm{BLIP}} \\
\left(10^{-17} \mathrm{~W} \mathrm{~Hz}^{-1 / 2}\right)\end{array}$ \\
\hline $1 \mathrm{~mm}$ long & 230 & 55 & 23 & 9 \\
$1 \mathrm{~mm}$ short & 290 & 45 & 23 & 10 \\
$860 \mu \mathrm{m}$ & 350 & 30 & 24 & 12 \\
$740 \mu \mathrm{m}$ & 405 & 20 & 22 & 13 \\
\hline
\end{tabular}

\section{ACKNOWLEDGEMENTS}

We gratefully acknowledge the support of NASA (grant NNG06GG16G), the NSF (grant AST-0705157) and the generous contributions of the Gordon and Betty Moore Foundation.

\section{REFERENCES}

[1] Puget, J.-L., Abergel, A., Bernard, J.-P., Boulanger, F., Burton, W.B., Desert, F.-X., \& Hartmann, D. "Tentative detection of a cosmic far-infrared background with COBE", A\&A, Vol. 308, L5-L8 (1996).

[2] Fixsen, D.J., Dwek, E., Mather, J.C., Bennett, C.L., \& Shafer, R.A. "The Spectrum of the Extragalactic Far-Infrared Background from the COBE FIRAS Observations," AJ, Vol. 508, pp. 123-128 (1998).

[3] Day, P.K., LeDuc, H.G., Mazin, B.A., Vayonakis, A., \& Zmuidzinas, J. “A broadband superconducting detector suitable for use in large arrays", Nature, Vol. 425, pp. 817-821 (2003).

[4] Goldin, A., Bock, J.J., Hunt, C., Lange, A.E., LeDuc, H.G., Day, P.K., Vayonakis, A., \& Zmuidzinas, J. "SAMBA: Superconducting antenna-coupled multi-frequency bolometric array", in Ninth International Workshop on Low Temperature Detectors, eds. F. S. Porter, D. McCammon, M. Galeazzi, and C. K. Stahle, AIP Conf. Proc. Vol. 605, pp. 251-254, New York: AIP (2002).

[5] Goldin, A., Bock, J.J., Hunt, C.L., Lange, A.E., LeDuc, H.G., Vayonakis, A., \& Zmuidzinas, J. "Design of broadband filters and antennas for SAMBA", Proc. SPIE Conference on Millimeter and Submillimeter Detectors for Astronomy, eds. T. G. Phillips and J. Zmuidzinas, Vol. 4855, pp. 163-171 (2003). 
[6] Mazin, B.A., Day, P.K., Irwin, K.D., \& Zmuidzinas, J. "Digital readouts for large microwave low-temperature detector arrays". Nucl. Instr. \& Meth. in Phys. Res., Vol. 559, pp. 799-801 (2006).

[7] Glenn, J., Ade, P.A.R, Amarie, M., Bock, J.J., Edgington, S.F., Goldin, A., Golwala, S., Haig, D., Lange, A.E., Laurent, G., Mauskopf, P.D., Yun, M., Nguyen, H. "Current status of Bolocam: a large-format millimeter-wave bolometer camera". Proc. SPIE Conference on Millimeter and Submillimeter Detectors for Astronomy, eds. T.G. Phillips \& J. Zmuidzinas, Vol. 4855, pp. 30-40 (2003).

${ }^{[8]}$ Haig, D.J., Ade, P.A.R., Aguirre, J.E., Bock, J.J., Edgington, S.F. Enoch, M.L., Glenn, J., Goldin, A., Golwala, S., Heng, K., Laurent, G., Maloney, P.R., Mauskopf, P.D., Rossinot, P., Sayers, J., Stover, P., \& Tucker, C. "Bolocam: status and observations". SPIE Conference on Millimeter and Submillimeter Detectors for Astronomy II, Eds. J. Zmuidzinas, W.S. Holland, \& S. Withington Vol. 5498, pp. 78-94 (2004).

[9] Laurent, G.T., Aguirre, J.E., Glenn, J., Ade, P.A.R., Bock, J.J., Edgington, S.F., Goldin, A., Golwala, S.R., Haig, D., Lange, A.E., Maloney, P.R., Mauskopf, P.D., Nguyen, H., Rossinot, P., Sayers, J., \& Stover, P. "The Bolocam Lockman Hole Submillimeter Galaxy Survey: Galaxy Candidates and Number Counts", ApJ, Vol. 623, pp. 742-762 (2005).

[10] Enoch, M.L., Young, K.E., Glenn, J., Evans, N.J. II, Golwala, S.R., Sargent, A.I., Harvey, P., J., Aguirre, J.E., Goldin, A., Haig, D., Lange, A., Laurent, G.T., Maloney, P., Mauskopf, P.D., Rossinot, P., \& Sayers, J. "Bolocam Survey for $1.1 \mathrm{~mm}$ Dust Continuum Emission in the c2d Legacy Clouds : I. Perseus". ApJ, Vol. 638, pp. 293-313 (2006).

[11] Sayers, J., Golwala, S.R., Rossinot, P., Ade, P.A.R., Aguirre, J.E., Bock, J.J., Edgington, S.F., Glenn, J., Goldin, A., Haig, D., Knowles, B., Lange, A.E., Laurent, G.T., Mauskopf, P.D., \& Nguyen, H.T. "A Search for Cosmic Microwave Background Anisotropies on Arcminute Scales with Bolocam", submitted to the ApJ (astro-ph arXiv:0805.3151v1).

[12] Schlaerth, J., Vayonakis, A., Day, P., Glenn, J., Golwala, S., Kumar, S., LeDuc, H., Mazin, B., Vaillancourt, J., \& Zmuidzinas, J. "A millimeter and submillimeter kinetic inductance detector camera", Journal of Low Temperature Physics, 151,684-689 (2008).

[13] Gao, J., Zmuidzinas, J., Mazin, B., LeDuc, H.G., \& Day, P.K. "Noise properties of superconducting coplanar waveguide microwave resonators”, Appl. Phys. Lett., 90,102507 (2007). 